\title{
Front propagation in a phase field model with phase-dependent heat absorption
}

\author{
Konstantin B. Blyuss *,1, Peter Ashwin, C. David Wright \\ School of Engineering, Computer Science and Mathematics \\ University of Exeter, Exeter, EX4 $4 Q E, U K$ \\ Andrew P. Bassom \\ School of Mathematics and Statistics, University of Western Australia \\ Crawley 6009, Australia
}

\footnotetext{
* Corresponding author.

Email address: blyuss@maths.ex.ac.uk (Konstantin B. Blyuss).

1 Tel.: +44 1392 263979; fax: +44 1392263997.
} 


\begin{abstract}
We present a model for the spatio-temporal behaviour of films exposed to radiative heating, where the film can change reversibly between amorphous (glassy) and crystalline states. Such phase change materials are used extensively in read-write optical disk technology.

In cases where the heat absorption of the crystal phase is less than that in the amorphous state we find that there is a bi-stability of the phases. We investigate the spatial behaviours that are a consequence of this property and use a phase field model for the spatio-temporal dynamics in which the phase variable is coupled to a suitable temperature field. It is shown that travelling wave solutions of the system are possible and, depending on the precise system parameters, these waves can take a range of forms and velocities. Some examples of possible dynamical behaviours are discussed and we show, in particular, that the waves may collide and annihilate. The longitudinal and transverse stability of the travelling waves are examined using an Evans function method which suggests that the fronts are stable structures.
\end{abstract}

Key words: Phase field, Phase change materials, Travelling fronts, Stability. 


\section{Introduction}

Phase field models are known to be an effective method for simulating the solidification of alloys $[8,13,15,33,34]$. The phase field itself is, in general, a function of both space and time, and quantifies the crystalline fraction present within a sample. Of the other physical processes present it is thought that the variation in temperature is the most important and coupled systems can be derived that relate the evolution of phase field to the temperature distribution. Such equations have been successfully used to study dendritic growth analytically and numerically during both isotropic and anisotropic solidification. More recently, phase field methods have been extended to describe the formation of grain boundaries during solidification $[16,18]$ - then the crystalline orientation in the solid phase must also be taken into account. Another area where phase field methods are known to be helpful is in reproducing physical results concerning systems with memory [11,24]. In this class of problem processes are typically characterised by some slow time relaxation and, as a result, the whole system becomes non-local in time.

Most mathematical studies of phase change processes in material used for optical data storage are currently based on the Johnson-Mehl-Avrami-Kolmogorov (JMAK) approach [14] in which it is supposed that crystallisation occurs uniformly in the whole sample of phase-change material. It is also assumed that this process is uniform in time, so stationary nucleation and growth rates can be used for modelling the crystallisation. However, recent experimental evidence suggests that both these simplifications are somewhat unrealistic for not only does the nucleation takes place mainly on the boundary between the phase-change material and the surrounding substrates [21,25], but there are typically non-negligible incubation times preceding the onset of crystallisation [32]. In recognition of these difficulties we have proposed a model based on the so-called 'master equation' approach $[5,29]$. In this framework the processes of crystallisation and/or melting are described by the time evolution of the density of crystal clusters. Even though this model has reproduced experimental results it has not yet explicitly incorporated any spatial distribution of the clusters. In this respect, a phase field method should be a suitable candidate for introducing spatial structure in an analysis of crystallisation within phase-change materials.

In this paper we concentrate on the use of a phase field approach in modelling fronts that can arise during the crystallisation of a phase-change alloy such as $\mathrm{Ge}_{2} \mathrm{Sb}_{2} \mathrm{Te}_{5}$ (GST), which is used as an active material for mass data storage and, in particular, for optical disk memory technology [17,31]. If this material is illuminated with a laser to its melting temperature and then cooled rapidly, the crystallisation process is quenched before nucleation and growth can occur; this results in the sample being transformed to an amorphous phase. 
Conversely, if the sample is heated to a temperature lower than the melting point (but before the nucleation cluster size gets too large) one can induce rapid growth of crystals in the sample. Since the material properties of the solid and amorphous phases are markedly different, the state of the system can be determined simply by illuminating it with a low power laser and measuring the reflected light, or by finding some other physical characteristic such as its conductivity.

Depending on the wavelength of the illuminating laser and the exact structure of the sample and its surroundings, the rate of absorption of heat from the laser varies with phase. In this work we consider some fundamental phenomena induced by this effect that might appear experimentally and should be controlled in the use of phase change materials for optical recording. To be specific, we incorporate illumination and phase-dependent absorption in the equation for the temperature variation, and this influences the phase change processes as described by the field equation. As it will be shown, depending on the values of parameters, the system will admit the crystallisation/melting fronts, which will have sharp interfaces and propagate with a constant velocity. Physically these fronts correspond to the situation when one phase completely invades the space occupied by another phase. Unlike many hydrodynamic systems, in the present situation the velocity of those fronts cannot be taken arbitrarily, but rather it is determined by the problem parameters.

An important question is the stability of these crystallisation/melting fronts. To study the linearized stability problem we use the so-called Evans function method. In this approach one introduces the Evans function as a complex analytic function associated with the linearization around the travelling wave, and its zeros correspond to the eigenvalues of the linearized stability problem. It was originally introduced by Evans [9], and later generalized by Alexander et al. [1]. The first numerical calculation of the Evans function was also performed by Evans [9], and subsequently followed by Swinton and Elgin [30] and Pego et al. [23]. There are two main problems associated with the calculation of the Evans function. The first one is that when the eigenvectors corresponding to, say, eigenvalues with negative real part, are integrated from some large $L_{\infty}$ to zero, one cannot numerically maintain their linear independence. Attempts to resolve this problem by using orthogonalization will lead to the loss of analyticity, as well as to the need to solve nonlinear equations. The second problem is that starting eigenvectors will not in general be analytic for all values of the spectral parameter. Both these problems can be circumvented by using exterior algebra and the compound matrix method introduced by $\mathrm{Ng}$ and Reid [20] for hydrodynamic stability problems. When using the compound matrix method, the solutions do not lie in a linear space, but rather on a Grassmanian manifold, and the numerical integration scheme should be chosen is such a way that it preserves this manifold. Allen and Bridges [2] have shown that the class of Gauss-Legendre Runge-Kutta (GL-RK) methods preserve the 

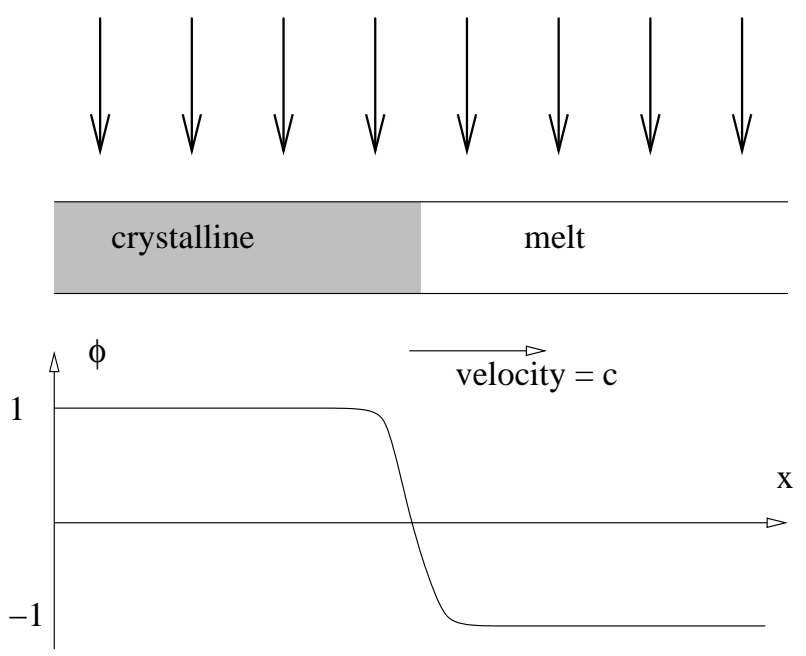

Fig. 1. Schematic diagram showing the modelling of the melting dynamics of a sample under uniform irradiation but phase-dependent absorption. The phase $\phi$ varies between -1 (melt) and 1 (solid) respectively.

Grassmanian $G_{2}\left(\mathbb{C}^{4}\right)$ to machine accuracy. This result motivates the particular choice of the numerical integrator in this paper.

The outline of the paper is as follows. In the next section the details of the model are introduced, and its elementary properties in terms of steady states and their stability are discussed in Section 3. In Section 4 numerical simulations are used to find crystallisation/melting fronts and investigate the dependence of their velocity on the problem parameters. Both the one- and two-dimensional stability of the frontal solutions are examined in Section 5 using the above-mentioned Evans function approach. The paper concludes with a summary of our principal findings together with a brief consideration of their implications.

\section{The mathematical model}

To begin, we consider a one-dimensional sample of homogeneous material occupying some region $\Omega$, and introduce the phase field $\phi(x, t)$; the convention adopted is that $\phi \in[-1,1]$ with the lower limit $\phi=-1$ corresponding to pure melt while $\phi=1$ represents solid. Figure 1 sketches the set-up we envisage; a sample of phase-change material is subjected to uniform radiative heating which can lead to the formation of a travelling solidification or melting front. Physically our domain corresponds to a one-dimensional slice of a two-dimensional crystal with the dynamics uniform in the perpendicular direction. 
The dynamics of phase change processes is based on the phase field equation used by Karma and Rappel [13] in their study of crystal growth:

$$
\partial_{t} \phi=-\partial_{\phi} f(\phi)-u \hat{\lambda} \partial_{\phi} g(\phi)+p^{2} \nabla^{2} \phi
$$

The solidification dynamics is governed by the functions $f$ and $g$ given by

$$
f(\phi)=-\frac{\phi^{2}}{2}+\frac{\phi^{4}}{4} \quad \text { and } \quad g(\phi)=\phi-\frac{2 \phi^{3}}{3}+\frac{\phi^{5}}{5}
$$

the interface thickness $p$ is a small parameter and $\hat{\lambda}$ is a measure of the strength of coupling between the phase field $\phi$ and a dimensionless temperature field $u$. More specifically, $u \equiv\left(T-T_{\mathrm{M}}\right) /\left(L / c_{P}\right)$ where $T$ is the temperature of the sample which melts when $T=T_{\mathrm{M}}, L$ is the latent heat of melting and $c_{p}$ is the specific heat at constant pressure. We remark that the time variable has been scaled so as to incorporate the characteristic time $\tau$ of attachment of atoms at the interface. Equation (1) can be thought of as a phenomenological balance driving the evolution of the system toward equilibrium states as given by the minima of free energy. To see this we notice that if one introduces a Helmholtz free energy

$$
\mathcal{F}=\int_{\Omega} d \mathbf{x}\left[\frac{p^{2}}{2}|\nabla \phi|^{2}+E(\phi, u)\right],
$$

with an energy density $E(\phi, u)=f(\phi)+\hat{\lambda} u g(\phi)$, then equation (1) can be recovered simply by setting [13]

$$
\phi_{t}=-\frac{\delta \mathcal{F}}{\delta \phi}
$$

which indicates that temporal dynamics of the phase field is indeed trying to minimise the free energy $\mathcal{F}$.

To complete the description of the system the phase field equation is augmented by one for the temperature evolution. In our case this has the form

$$
\partial_{t} T=\left[a_{1}+a_{-1}+\left(a_{1}-a_{-1}\right) \phi\right] \frac{I}{2}+b\left(T_{a}-T\right)+d \nabla^{2} T+\delta \partial_{t} \phi / 2,
$$

where $a_{ \pm 1}$ are the radiative absorption coefficients for the solid and molten phases, $b$ is a thermal emission coefficient, $T_{a}$ is the ambient temperature $\left(T_{a} \ll T_{\mathrm{M}}\right)$, $d$ denotes a thermal diffusivity, $\delta=L / c_{p}$ and $I$ is the rate of incident heating. The last term in (3) corresponds to the latent heat production at the interface [13] and, under the assumption that the energy throughput of 
the system is large compared to the latent heat and changes in specific heat, it can be safely omitted to give

$$
\partial_{t} T=\left[a_{1}+a_{-1}+\left(a_{1}-a_{-1}\right) \phi\right] \frac{I}{2}+b\left(T_{a}-T\right)+d \nabla^{2} T
$$

Equations (1) and (4) together with appropriate boundary conditions form a closed system for the phase change processes that occur in a sample of material when uniformly irradiated by a laser.

\section{$3 \quad$ Steady states}

We begin our analysis by studying spatially uniform steady states of the system $(1,4)$. These are given as solution pairs $(\phi, T)$ of the nonlinear problem

$$
\begin{aligned}
& \phi\left(1-\phi^{2}\right)-\tilde{\lambda}\left(T-T_{M}\right)\left(1-\phi^{2}\right)^{2}=0, \\
& \frac{1}{2}\left[a_{1}+a_{-1}+\left(a_{1}-a_{-1}\right) \phi\right] I+b\left(T_{a}-T\right)=0,
\end{aligned}
$$

where $\tilde{\lambda} \equiv c_{p} \hat{\lambda} / L$. The second of these equations is linear and so it is simple to relate the equilibrium phase to the temperature as

$$
T=T_{a}+\left[a_{1}+a_{-1}+\left(a_{1}-a_{-1}\right) \phi\right] \frac{I}{2 b} .
$$

System (5) admits equilibria

$$
(\phi, T)=\left(-1, T_{-1}\right) \quad \text { and } \quad(\phi, T)=\left(1, T_{1}\right)
$$

where

$$
T_{ \pm 1}=T_{a}+\frac{a_{ \pm 1} I}{b}
$$

From the physical point of view these equilibria correspond to the situations when the system consists totally of either melt or of solid.

Experimentally the relation between absorption coefficients of the molten and solid phases can be altered by changing the stoichiometry of the materials involved or by changing the multi-layer structure of the optical disc [37]. In 
particular, ii is possible to achieve the situation in which the heat absorption coefficient of the melt exceeds that of a solid:

$$
a_{-1}>a_{1}
$$

In this case there is a bi-stability between the solid/melt states. For the remainder of the paper we assume the relation (8) between absorption coefficients of melt/solid phases holds. From this relation one can find that for certain range of intensities $I$ it is possible to satisfy the inequality

$$
\frac{a_{-1} I}{b}>T_{\mathrm{M}}-T_{a}>\frac{a_{1} I}{b}
$$

or in other words, $T_{1}<T_{\mathrm{M}}<T_{-1}$ which implies a physically realistic situation when the equilibrium temperature of melt exceeds the melting temperature $T_{\mathrm{M}}$, while the equilibrium temperature of the solid state is lower than $T_{\mathrm{M}}$. Notice also that the particular form of equation (1) means that the stability of the steady states $\left( \pm 1, T_{ \pm 1}\right)$ does not depend on the actual relation between absorption coefficients nor on the intensity $I$ of external irradiation.

Assuming $\phi \neq \pm 1$ one can reduce (5a) to a cubic equation for the equilibrium phase:

$$
\phi-\tilde{\lambda}(A+B \phi)\left(1-\phi^{2}\right)=0
$$

where

$$
A=\frac{T_{1}+T_{-1}}{2}-T_{\mathrm{M}} \quad \text { and } \quad B=\frac{T_{1}-T_{-1}}{2} .
$$

It follows that $B<0$ and $|A| \leq|B|$ and if we introduce the quantities

$$
\alpha=\frac{A^{2} \tilde{\lambda}-3 B(1-B \tilde{\lambda})}{9 B^{2} \tilde{\lambda}} \quad \beta=\frac{2 A^{2} \tilde{\lambda}-9 B-18 B^{2} \tilde{\lambda}}{27 B^{3} \tilde{\lambda}}
$$

then equation (10) has three real roots $\phi_{1}-\phi_{3}$ given by

$$
\begin{aligned}
& \phi_{1}=-\frac{A}{3 B}+\frac{2}{3 B} \sqrt{\frac{A^{2} \tilde{\lambda}-3 B(1-B \tilde{\lambda})}{\tilde{\lambda}}} \sin \psi, \\
& \phi_{2,3}=-\frac{A}{3 B} \mp \frac{2}{3 B} \sqrt{\frac{A^{2} \tilde{\lambda}-3 B(1-B \tilde{\lambda})}{\tilde{\lambda}}} \sin \left(\frac{\pi}{3} \pm \psi\right),
\end{aligned}
$$


where the angle $\psi$ satisfies $2 \alpha^{3 / 2} \sin (3 \psi)=\beta$ and $|\psi| \leq \pi / 6$. Depending on the precise relation between $A, B$ and $\tilde{\lambda}$ there can be one or more roots with $\phi \in[-1,1]$, which, from the physical point of view, are the ones of interest.

The additional steady states which do not correspond to either pure melt or pure solid are referred to as "mushy layers" and these have been used to model a porous region made up of crystals surrounded by residual melt $[28,35,36]$. A mushy layer separates the region occupied by the completely solidified material from that occupied by the melt phase. Mushy layers may form as a result of the morphological instability of a solidification front [19] and occur frequently during the solidification of binary and ternary alloys [3]. The presence of a mushy layer during solidification can greatly influence the transport of heat, mass and solute, and therefore the final solidified product. Within mushy layers a local thermodynamic equilibrium prevails, and this can be maintained experimentally on a time scale of a few seconds for metallic mushy layers [10]. For our system we can interpret the steady states with the solid fraction $\sigma$ (defined as $(1+\phi) / 2$ ) not equal to 0 or 1 , as the states of mushy layer, where the transition from melt to solid takes place.

To examine the stability of the steady states $(\hat{\phi}, \hat{T})$ we linearise system $(1,4)$ around $(\hat{\phi}, \hat{T})$ and look for solutions in the form

$$
(\phi(x, t), T(x, t))=(\hat{\phi}, \hat{T})+\left(C_{1}, C_{2}\right) \exp (i k x+\mu t)
$$

with $C_{1,2}$ constants. This leads to the dispersion relation

$$
\begin{aligned}
& \mu^{2}+\mu\left[b+\left(d+p^{2}\right) k^{2}-1+3 \hat{\phi}^{2}-4 \tilde{\lambda} \hat{\phi}\left(\hat{T}-T_{\mathrm{M}}\right)\left(1-\hat{\phi}^{2}\right)\right] \\
& +\frac{1}{2} \tilde{\lambda}\left(1-\hat{\phi}^{2}\right)^{2}\left(a_{1}-a_{-1}\right) I+p^{2} k^{2}\left(b+d k^{2}\right)- \\
& \left(b+d k^{2}\right)\left[1-3 \hat{\phi}^{2}+4 \tilde{\lambda} \hat{\phi}\left(\hat{T}-T_{\mathrm{M}}\right)\left(1-\hat{\phi}^{2}\right)\right]=0
\end{aligned}
$$

from which it follows readily that already linearly stable steady states are further stabilised by diffusion. However, this does not exclude the possibility of existence of linearly unstable equilibria, which would correspond to the mushy states.

\section{Travelling wave solutions}

To examine the properties of crystallisation fronts it is convenient to look for solutions of $(1,4)$ in the form of travelling waves

$$
\phi=\psi(z), \quad T=S(z), \quad z \equiv x-c t
$$



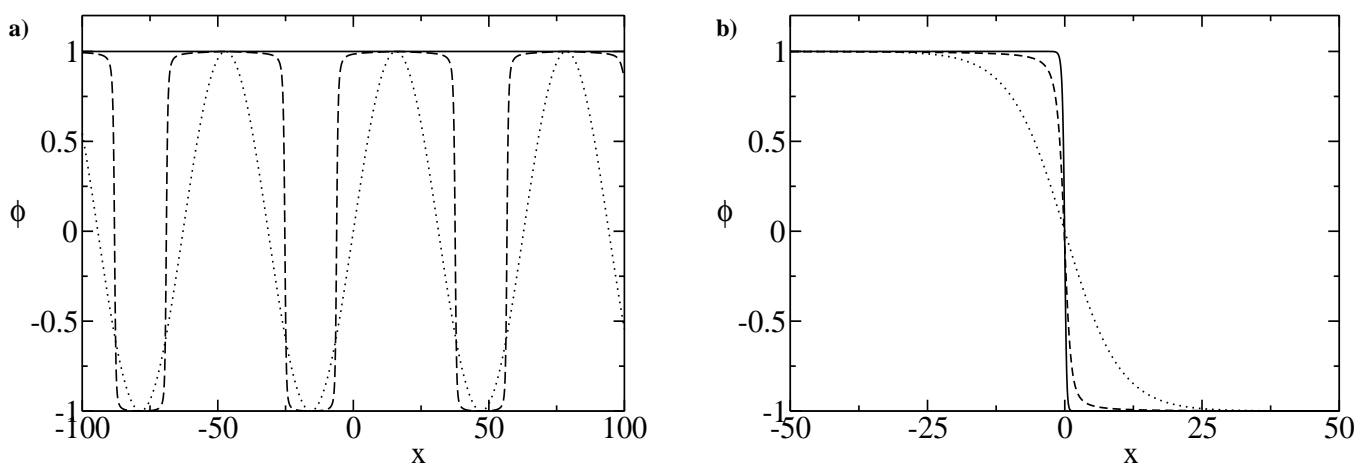

Fig. 2. Shape of crystallisation fronts in the system $(1,4)$ with the parameters $a_{1}=4, a_{-1}=6, I=1.2, b=1, \lambda=1, p=0.316, d=1, T_{a}=2.9$ and $T_{\mathrm{M}}=9.8$ at $t=0$ (dotted), $t=0.5$ (dashed) and $t=10$ (solid). a) Sinusoidal initial condition Eq. (16). b) Initial condition given by Eq. (17).

The substitution of these expressions into the governing system yields the fourth-order problem

$$
\begin{aligned}
& p^{2} \psi^{\prime \prime}+c \psi^{\prime}+\left[\psi-\tilde{\lambda}\left(S-T_{\mathrm{M}}\right)\left(1-\psi^{2}\right)\right]\left(1-\psi^{2}\right)=0, \\
& d S^{\prime \prime}+c S^{\prime}+\left[a_{1}+a_{-1}+\left(a_{1}-a_{-1}\right) \psi\right] \frac{I}{2}+b\left(T_{a}-S\right)=0
\end{aligned}
$$

where the prime denotes a derivative with respect to $z$. Two equilibria exist: these are $(\psi, S)=\left( \pm 1, T_{ \pm 1}\right)$ and a travelling wave represents a heteroclinic connection between them. The linearization of the system (15) near either of the equilibria leads to a spectrum

$$
\operatorname{spec}(A)=\left\{-\frac{c}{2 p^{2}} \pm \frac{1}{2 p^{2}} \sqrt{c^{2}+8 p^{2}},-\frac{c}{2 d} \pm \frac{1}{2 d} \sqrt{c^{2}+4 b d}\right\}
$$

and it follows that the dimensions of the stable and unstable manifolds of the equilibria are $\operatorname{dim} W^{u}\left(1, T_{1}\right)=\operatorname{dim} W^{s}\left(-1, T_{-1}\right)=2$. Hence, generically these two manifolds do not intersect in a four-dimensional ambient space, and the heteroclinic connections exist only for isolated values of velocity $c$, which is determined by the model parameters.

In order to obtain insight into the possible existence of various travelling waves for different values of parameters, we solved the system $(1,4)$ numerically using the Fortran NAG routine D03PCF. Since we are looking for travelling waves, the numerical domain of integration was taken to be large compared to the front width and typically the system was solved for $x \in[-L, L]$ with $L \approx 100$. For simplicity Neumann conditions were imposed at the ends of the domain but the results shown are not sensitive to the exact forms of any physically sensible boundary conditions. 

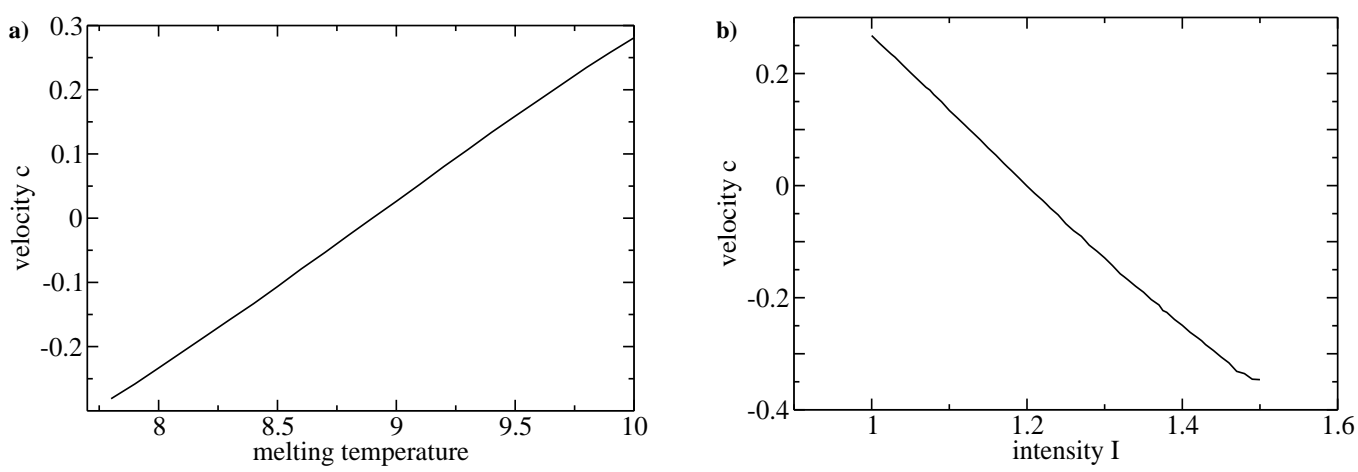

Fig. 3. Variation in the velocity $c$ of front solutions of system $(1,4)$ with parameters chosen as $a_{1}=4, a_{-1}=6, b=1, \lambda=1, p=0.316, d=1$ and $T_{a}=2.9$. a) Changing melting temperature $T_{\mathrm{M}}$ with fixed light intensity $I=1.2$. b) Changing light intensity $I$ with fixed melting temperature $T_{\mathrm{M}}=9.8$.

As a first example we present in Fig. 2a) the results of simulations when initial condition was taken to be a simple periodic function of the form

$$
\phi(x, 0)=\sin (x / 10), T(x, 0)=\frac{T_{1}+T_{-1}}{2}+\frac{T_{-1}-T_{1}}{2} \sin (x / 10) .
$$

What one observes is an initial steepening of the sine profile, which eventually develops into shock-like structures. Neighbouring fronts then start propagating in opposite directions which results in annihilation and consequent disappearance of any wave structure. In this case the system settles on a uniform steady state corresponding to solid everywhere.

To illustrate the evolution of $(1,4)$ from a profile which approaches the steady states $\left(1, T_{1}\right)$ and $\left(-1, T_{-1}\right)$ as $x \rightarrow-\infty$ and $x \rightarrow \infty$, we next consider the initial condition

$$
\phi(x, 0)=-\tanh (x / 10), T(x, 0)=\frac{T_{1}+T_{-1}}{2}+\frac{T_{-1}-T_{1}}{2} \tanh (x / 10) .
$$

In the simulations whose results are shown in Fig. 2b) we broke the symmetry between the two steady states by choosing the melting temperature $T_{\mathrm{M}}$ to be closer to the equilibrium temperature $T_{-1}$ of the molten phase than its value $T_{1}$ for the solid. As before, the solution initially steepens and quickly reaches the state characterised by a sharp interface separating the phases. This solution then propagates without change of shape. In this case the solutions are the crystallisation fronts (also called solidification fronts), i.e. the crystallised phase invades the space of the molten phase.

With the same values of parameters but now with the melting temperature chosen to be closer to that of the solid phase, then the same initial conditions will result in melting fronts; that are fronts that propagate in the opposite 
direction. Figure 3 shows the effects on the front velocity of varying the melting temperature and light intensity. (Negative values of velocity correspond to the fronts propagating in the negative $x$-direction.) Figure $3 \mathrm{~b}$, which relates to a fixed $T_{\mathrm{M}}=9.8$ and varying laser intensity $I$, suggests that the velocity decreases almost linearly with increasing $I$ until some maximum intensity is reached; the existence of such a greatest $I$ is evident from the form of conditions (9). For fixed values of parameters there comes a point when one of these inequalities must be violated. Another interesting symmetry which directly follows from the equations $(1,4)$ is that changing $x \rightarrow-x$, and also changing the sign of velocity $c \rightarrow-c$ leaves this system invariant. This means that whenever system $(1,4)$ admits crystallisation fronts going to the right, it also admits crystallisation fronts propagating to the left.

\section{$5 \quad$ The stability of travelling waves}

After the existence of travelling front solutions has been established for some parameter values, the next important question to be answered is that of their stability. Unfortunately, this question is not clear-cut for there exist several possible definitions of travelling wave stability, each reflecting some particular characteristic of the problem. Among the most often studied is the issue of linear stability which reflects the absence of any eigenvalues with positive real part in the spectrum of the linearization near the travelling wave. A more advanced approach is that of nonlinear, or Lyapunov, stability, which means that in a properly defined functional space there exists a class of initial conditions which tend to the travelling wave solution as time goes to infinity. A modern review of stability of travelling waves with an emphasis on dynamical systems approaches can be found in Sandstede [27].

In this work we concentrate on linear stability methods and consider two types of perturbations: longitudinal and transverse. The former represent physically small changes in the shape of fronts which travel in the same direction as the fronts themselves. The transverse perturbations correspond to a perturbation whose direction of propagation is different from (and possibly orthogonal to) the basic direction of front motion. As the method of choice for the analysis of linear stability we use the Evans function approach mentioned in the introduction. This is a dynamical systems formulation of the stability problem which makes use of a complex valued function, called the Evans function, whose zeros correspond to the isolated eigenvalues of the linearization operator. The Evans function method has been successfully used to study linear longitudinal and transverse stability of solitary waves in various hydrodynamical contexts $[6,7]$ and in systems describing the dynamics of chemical reactions [4] or combustion waves [12]. 


\subsection{Longitudinal stability}

We start by considering the one-dimensional linear stability of the travelling waves found earlier. Let solution of the system $(1,4)$ be

$$
(\phi(x, t), T(x, t))=(\psi(z), S(z))+\operatorname{Re}\{\exp (\mu t)(\tilde{\phi}(z), \tilde{T}(z))\}
$$

where $\psi(z)$ and $S(z)$ are the travelling waves from the previous section. Substituting this into $(1,4)$ and linearising gives, after dropping the tildes,

$$
\begin{aligned}
& p^{2} \phi^{\prime \prime}+c \phi^{\prime}+\left[1-\mu-3 \psi^{2}(z)\right] \phi+P(z) \phi-\tilde{\lambda} T\left[1-\psi^{2}(z)\right]^{2}=0, \\
& d T^{\prime \prime}+c T^{\prime}=(b+\mu) T-\frac{1}{2}\left(a_{1}-a_{-1}\right) I \phi
\end{aligned}
$$

where the auxiliary function $P(z)$ is defined by

$$
P(z)=4 \tilde{\lambda} \psi(z)\left(S(z)-T_{\mathrm{M}}\right)\left[1-\psi^{2}(z)\right] .
$$

The travelling wave $(\psi(z), S(z))$ is linearly unstable if one can find a bounded solution of the system (19) with associated growth rate $\mu$ having positive real part. In order to tackle this problem we rewrite the system (19) as a first-order system

$$
\mathbf{v}_{z}=\mathbf{A}(z, \mu) \mathbf{v}
$$

where $\mathbf{v}=\left(\phi, \phi_{z}, T, T_{z}\right)^{T}$ and $\mathbf{A}(z ; \mu)$ is given by

$$
\mathbf{A}(z ; \mu)=\left(\begin{array}{cccc}
0 & 1 & 0 & 0 \\
a_{21} & -c / p^{2} & a_{23} & 0 \\
0 & 0 & 0 & 1 \\
\left(a_{-1}-a_{1}\right) I / 2 d & 0 & (b+\mu) / d & -c / d
\end{array}\right)
$$

where $a_{21} \equiv\left[\mu+3 \psi^{2}(z)-1-P(z)\right] / p^{2}$ and $a_{23} \equiv \tilde{\lambda}\left(1-\psi^{2}(z)\right)^{2} / p^{2}$. In the limit $z \rightarrow \pm \infty$ this matrix reduces to a constant form $\mathbf{A}_{\infty}(\mu) \equiv \lim _{z \rightarrow \pm \infty} \mathbf{A}(z, \mu)$ with eigenvalues

$$
\operatorname{spec}\left(\mathbf{A}_{\infty}\right)=\left\{ \pm \frac{\sqrt{\mu+2}}{p}, \pm \frac{\sqrt{d(b+\mu)}}{d}\right\}
$$


This spectrum implies there is a two-dimensional subspace of solutions $\mathbf{U}^{+}(z, \mu)$ of the equation (20) that decay exponentially as $z \rightarrow+\infty$ and another two-dimensional subspace of solutions $\mathbf{U}^{-}(z, \mu)$ that decay exponentially as $z \rightarrow-\infty$. If these two subspaces have a non-trivial intersection for some value of $\mu$, then this is an eigenvalue of the problem (20).

One can define the Evans function as [1]

$$
E(\mu)=\exp \left(-\int_{0}^{z} \tau(x, \mu) d x\right) \mathbf{U}^{-}(z, \mu) \wedge \mathbf{U}^{+}(z, \mu)
$$

where $\wedge$ is the wedge product, and

$$
\tau(z, \mu)=\operatorname{Tr}[\mathbf{A}(z, \mu)]
$$

Zeros of the Evans function correspond to the intersection of the abovementioned subspaces, and the requisite $\mu$ are then the eigenvalues of the linearised stability problem (19). In calculating the Evans function we follow the numerical approach developed by Bridges, Derks and Gottwald [7] in the context of the fifth-order KdV equation. To be more specific, we use the compound matrices to describe the dynamics of the subspaces $\mathbf{U}^{-}$and $\mathbf{U}^{+}$, as well as the Hodge star operator to match these solutions at $z=0$. Also, since our problem possesses a 2-2 splitting of eigenvalues at infinity, we have used the implicit midpoint rule to perform the time integration as for such splitting it preserves the Grassmanian manifold to machine accuracy [2].

For the purposes of numerical calculations one notices that $\mathbf{U}^{+}(z ; \mu)$ and $\mathbf{U}^{-}(z ; \mu)$ can be thought of as paths in a wedge space $\Lambda^{2}\left(\mathbb{C}^{4}\right)$ of two-forms on a complex four-dimensional space, which can be identified with $\mathbb{C}^{6}$. Their dynamics can hence be determined by integrating the system

$$
\frac{d}{d z} \mathbf{U}=\mathbf{A}^{(2)}(z, \mu) \mathbf{U}, \quad \mathbf{U} \in \bigwedge^{2}\left(\mathbb{C}^{4}\right)
$$

where the induced matrix $\mathbf{A}^{(2)}: \bigwedge^{2}\left(\mathbb{C}^{4}\right) \rightarrow \bigwedge^{2}\left(\mathbb{C}^{4}\right)$ is defined on a decomposable 2-form $\mathbf{x}=\mathbf{x}_{1} \wedge \mathbf{x}_{2}$ as $\mathbf{A}^{(2)} \mathbf{x}:=\mathbf{A} \mathbf{x}_{1} \wedge \mathbf{x}_{2}+\mathbf{x}_{1} \wedge \mathbf{A} \mathbf{x}_{2}$, see [2].

One can introduce the limiting matrix $\mathbf{A}_{\infty}^{(2)}(\mu)$ defined by

$$
\mathbf{A}_{\infty}^{(2)}(\mu)=\lim _{z \rightarrow \pm \infty} \mathbf{A}^{(2)}(z, \mu)
$$

and then for $\operatorname{Re}(\mu)>0$ the matrix $\mathbf{A}_{\infty}^{(2)}(\mu)$ has an eigenvalue $\sigma_{+}(\mu)$, which is the sum of two eigenvalues of $\mathbf{A}(\mu)$ having negative real part. This eigenvalue 
$\sigma_{+}(\mu)$ is simple, is an analytic function of $\mu$ and corresponds to an exponentially decaying behaviour at $z \rightarrow+\infty$, [2]. Similarly there exists an eigenvalue $\sigma_{-}(\mu)$, which corresponds to an exponentially decaying behaviour at $z \rightarrow-\infty$, and which is the sum of the two eigenvalues of $\mathbf{A}(\mu)$ with non-negative real part. If $\zeta^{ \pm}(\mu)$ are the eigenvectors associated with $\sigma_{ \pm}(\mu)$ so

$$
\mathbf{A}_{\infty}^{(2)}(\mu) \zeta^{ \pm}(\mu)=\sigma_{ \pm}(\mu) \zeta^{ \pm}(\mu)
$$

then the solutions $\mathbf{U}$ of the system (25) have the property $\lim _{z \rightarrow \pm \infty} \mathbf{U}(z ; \mu)=$ $\zeta^{ \pm}(\mu)$.

In parallel to system (25) one can also consider the adjoint problem

$$
\frac{d}{d z} \mathbf{V}=-\left[\mathbf{A}^{(2)}(z, \mu)\right]^{T} \mathbf{V}, \quad \mathbf{V} \in \stackrel{2}{\bigwedge}^{2}\left(\mathbb{C}^{4}\right)
$$

and identify the most unstable solution $\mathbf{V}^{-}$at $z=-\infty$ of this equation with the most unstable solution $\mathbf{U}^{-}$of the linearised system (25) at $z=$ $-\infty$. The asymptotic behaviours of such solutions is governed by the relation $\lim _{z \rightarrow-\infty} \exp \left(\overline{\sigma_{+}(\mu)} z\right) \mathbf{V}^{-}(z, \mu)=\eta^{-}(\mu)$, where $\eta^{-}(\mu)$ is the eigenvector of the matrix $-\overline{\mathbf{A}}^{T}$ associated with the eigenvalue $-\overline{\sigma_{+}}$, see $[2,7]$.

In order to simplify the numerical implementation, the inner product on $\wedge^{2}\left(\mathbb{C}^{4}\right)$ can be identified with the inner product $\langle\cdot, \cdot\rangle_{6}$ on $\mathbb{C}^{6}$, and then the Evans function may be written as [7]

$$
E(\mu)=\left\langle\overline{\mathbf{V}^{-}(0, \mu)}, \mathbf{U}^{+}(0, \mu)\right\rangle_{6}
$$

This function is approximated by integrating the equation

$$
\frac{d}{d z} \widetilde{\mathbf{U}}^{+}=\left[\mathbf{A}^{(2)}(z, \mu)-\sigma_{+}(\mu) \mathbf{I}_{d}\right] \widetilde{\mathbf{U}}^{+},\left.\quad \widetilde{\mathbf{U}}^{+}\right|_{z=L_{\infty}}=\zeta^{+}(\mu)
$$

with an implicit midpoint rule from $z=L_{\infty}\left(L_{\infty} \gg 1\right)$ to $z=0$. (Note that the scaling $\widetilde{\mathbf{U}}^{+}(z, \mu)=\exp \left(-\sigma_{+}(\mu) z\right) \mathbf{U}^{+}(z, \mu)$ removes the exponential growth.) For $z<0$ we integrate the adjoint equation

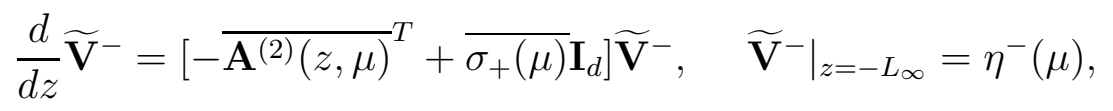

from $z=-L_{\infty}$ to $z=0$ with the corresponding $\widetilde{\mathbf{V}}^{-}(z, \mu)=\exp \left(\overline{\sigma_{+}(\mu)} z\right) \mathbf{V}^{-}(z, \mu)$ The eigenvectors of the limiting matrix $\mathbf{A}_{\infty}^{(2)}$ are normalised as $\left\langle\eta^{-}(\mu), \zeta^{+}(\mu)\right\rangle=$ 

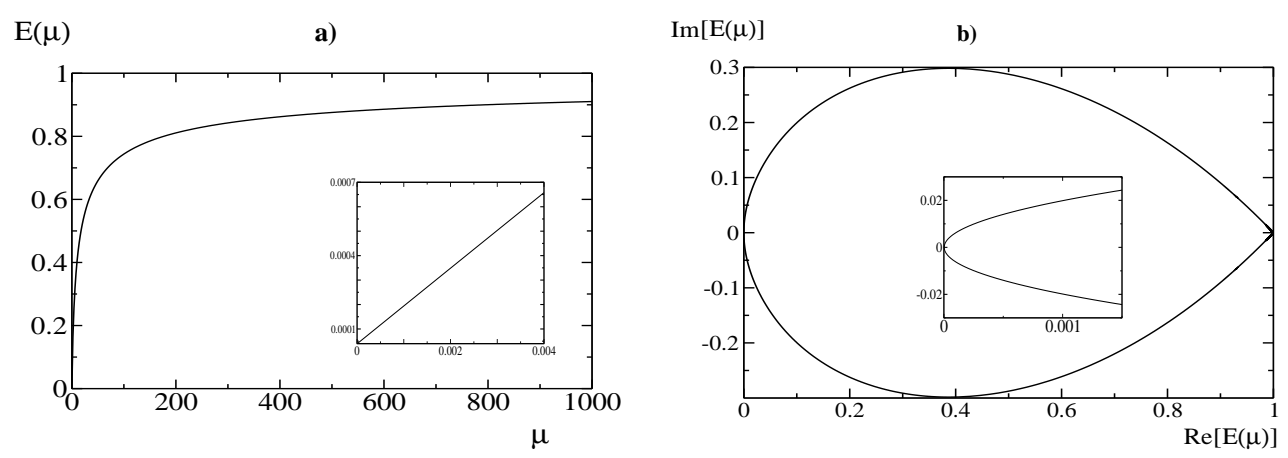

Fig. 4. (a). Evans function $E(\mu)$ evaluated along the real $\mu$ axis. (b). The real versus imaginary parts of the Evans function $E(\mu)$ for $\mu$ varying parallel to the imaginary axis with the small offset $\varepsilon=10^{-8}$. (The insert shows the local behaviour near the origin.)

1. At $z=0$, the Evans function now admits an equivalent form

$$
E(\mu)=\left\langle\overline{\mathbf{V}^{-}(\mathbf{0}, \mu)}, \mathbf{U}^{+}(0, \mu)\right\rangle_{6}=\left\langle\overline{\left\langle\overline{\mathbf{V}}^{-}(0, \mu)\right.}, \widetilde{\mathbf{U}}^{+}(0, \mu)\right\rangle_{6} .
$$

Results of the Evans calculations are presented in Fig. 4 for the travelling fronts found in the previous section. All the parameters are as before except for the melting temperature which is chosen to be $T_{\mathrm{M}}=9.8$. In this case the system $(1,4)$ possesses a solution in the form of a crystallisation front moving to the right with a constant velocity, which can be found numerically as $c \approx 0.23418$.

First, we perform calculations along the real $\mu$ axis. In this case, the Evans function itself is also real and, as Figure 4a shows, it asymptotically approaches a constant value of $E(\mu)=1$ as $\mu \rightarrow \infty$ (this value is determined by the abovementioned normalisation of eigenvectors of $\left.\mathbf{A}_{\infty}^{(2)}(\mu)\right)$. Numerical results show that starting with the value $E(0)=0$, the Evans function then monotonically increases and never crosses zero again. This suggests that most likely the linearised stability problem (19) does not have any real positive eigenvalues.

To conclude the linear stability analysis one has to investigate the possibility of complex eigenvalues having positive real part. In this case we evaluate the Evans function on varying the spectral parameter $\mu$ parallel to imaginary axis with a (small) offset $\mu=\varepsilon+i \tilde{\mu}$ to avoid the pole of the Evans function at $\mu=0$. Typically, $\tilde{\mu}$ was varied from 0 to $10^{8}$. By Cauchy's theorem, the winding number of a closed curve in the $\mu$ plane gives the number of unstable eigenvalues. Figure $4 \mathrm{~b}$ shows the results of the calculation in this case. The close-up near zero indicates that the winding number is zero, and therefore we conclude the travelling front solutions connecting the melt and solid equilibria are linearly stable. Even though we have studied numerically only a finite region of the complex plane, having eigenvalues beyond the half-circle of the radius $10^{8}$ would correspond to the physically unrealistic growth rates of the 


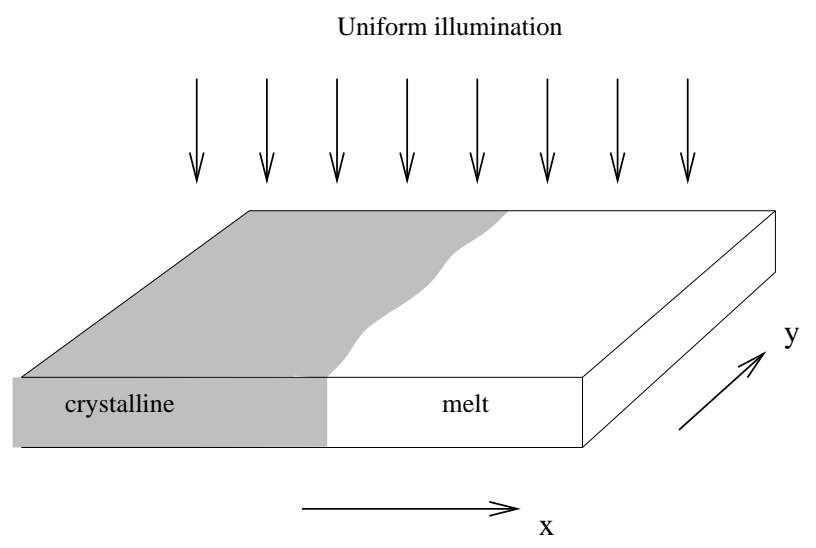

Fig. 5. Schematic diagram showing the melting dynamics in 2D under uniform irradiation but phase-dependent absorption. We examine the stability of the plane travelling wavefront to perturbations in the $y$ direction.

perturbation, and this suggests that most probably the stability picture is now complete.

We have also performed numerical simulations of the full system $(1,3)$ which includes the latent heat production. These simulations show that the system is robust with respect to the increase in the latent heat and retains the same features as in the case $\sigma=0$, namely, that initial profiles rapidly develop into sharp-interface fronts which then propagate with constant velocity.

\subsection{Transverse stability}

In the previous subsection we showed that the crystallisation fronts connecting the steady states of $(1,4)$ corresponding to the fully molten and fully crystallised states are linearly stable. From the physical point of view, it is also of interest to investigate the stability of the fronts with respect to transverse perturbations, i.e. perturbations in the direction orthogonal to that of front propagation. Figure 5 illustrates the two-dimensional set-up we consider. To model this case we replace Laplacian operators in the equations $(1,4)$ by their two-dimensional counterparts: $\nabla^{2} \rightarrow \partial_{x x}+\partial_{y y}$.

Now one can seek solutions of the system $(1,4)$ in the form

$$
(\phi(x, y, t), T(x, y, t))=(\psi(z), S(z))+\operatorname{Re}\{\exp (i k y+\mu t)(\tilde{\phi}(z), \tilde{T}(z))\}
$$

where $z \equiv x-c t$ is the travelling wave argument, $k$ is the transverse wavenumber, $\mu$ is the instability growth rate and $\psi(z)$ and $S(z)$ represent the onedimensionally stable front analysed in the previous sections. Substituting the 

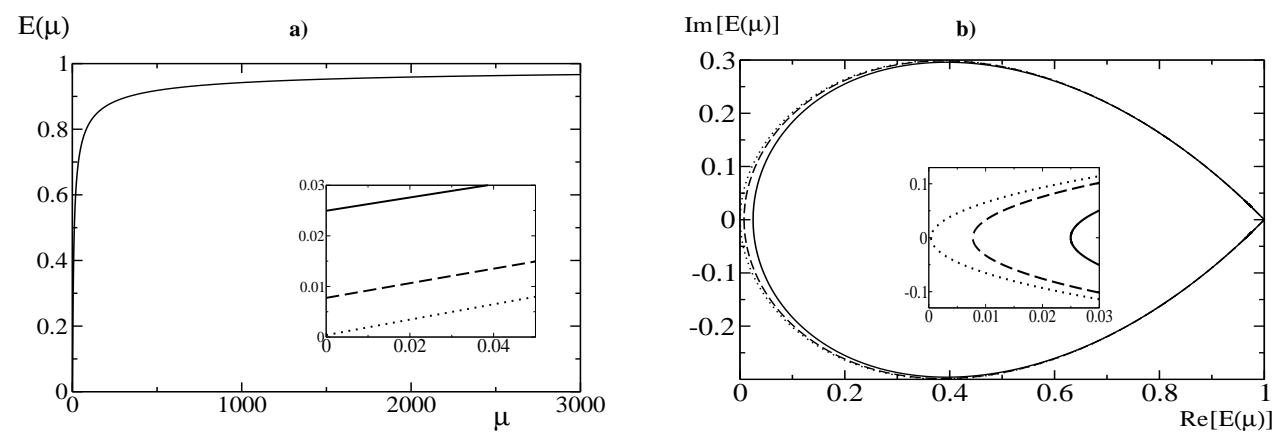

Fig. 6. Stability of crystallisation fronts with transverse wavenumbers $k=0.1$ (dotted), $k=0.5$ (dashed) and $k=1$ (solid). (a). Evans function $E(\mu)$ along real $\mu$ axis. (b). The real versus imaginary parts of the Evans function $E(\mu)$ for $\mu$ varying parallel to the imaginary axis with the small offset.

above expressions into $(1,4)$ and linearising, one obtains (after dropping the tildes)

$$
\begin{aligned}
& p^{2} \phi^{\prime \prime}+c \phi^{\prime}+\left[1-\mu-3 \psi^{2}(z)-k^{2} p^{2}\right] \phi+P(z) \phi-\tilde{\lambda} T\left[1-\psi^{2}(z)\right]^{2}=0 \\
& d T^{\prime \prime}+c T^{\prime}=\left(b+\mu+k^{2} d\right) T-\frac{\left(a_{1}-a_{-1}\right)}{2} I \phi .
\end{aligned}
$$

The one-dimensional travelling fronts are linearly transversely unstable if one can find bounded solutions of the system $(32)$ with $\operatorname{Re}(\mu)>0$ for some transverse wavenumber $k$. In order to analyse this problem we again use Evans functions. It is easy to see that the reformulation of (32) as a first-order system would coincide with (20) but for the two entries $a_{21}$ and $a_{43}$ in the matrix A which transform into $a_{21} \rightarrow a_{21}+k^{2}$ and $a_{43} \rightarrow a_{43}+k^{2}$. The inclusion of these extra terms proportional to $k^{2}$ does not affect the spectrum of $\mathbf{A}_{\infty}$ so the same construction of the Evans function as before is sufficient, save for the fact that now it also depends on the transverse wavenumber $k$ as an extra parameter, i.e. we have $E(\mu, k)$. In order to investigate the possibility of transverse instability, we increase $k$ starting from 0 , and for each fixed $k$ the Evans function $E(\mu, k)$ is evaluated in the complex $\mu$-plane. If for some value of $\mu$ it crosses zero, then this value is the eigenvalue of the linearised stability problem corresponding to a transverse wavenumber $k$.

Figure 6 shows the plots of the Evans function as a function of a spectral parameter $\mu$ when integrated along the real $\mu$ axis and also along the line parallel to the imaginary axis in order to investigate the possibility of complex eigenvalues with positive real part. The value of the transverse wavenumber $k$ was varied to study both the long- and the short-wavelength transverse instability. Figure 6a illustrates the Evans function evaluated along the real axis of spectral parameter $\mu$. The qualitative behaviour is the same as in the one- 
dimensional case: the Evans function increases monotonically and approaches its value of unity as $\mu \rightarrow \infty$. At the same time, it is noteworthy that the value of $E(0)$ increases with $k \neq 0$, emphasising the fact that the one-dimensional front does not solve the full problem with the transverse direction included. This fact can also be heuristically understood by inspecting the linearized stability problem (32) and observing that in this system as compared with (19), the eigenvalues $\mu$ have been substituted by $\mu+k^{2} p^{2}$ in the first equation and $\mu+d k^{2}$ in the second equation. From here one can expect that if the eigenvalues $\mu$ are in the left half-plane in the longitudinal case, in the case of transverse perturbations they will have even more negative real part if $k>0$. The plot of the Evans function in the complex domain of the spectral parameter is shown in Fig. 6b and it confirms the absence of eigenvalues in the complex right half-plane. This implies that the crystallisation fronts are persistent against perturbations aligned away from the basic direction of their propagation.

\section{Conclusions}

In this paper we have used a phase field model to investigate the effect of phasedependent heat absorption on a dynamics in a phase change sample irradiated by a laser. This model shows that the phase-dependent heat absorption is sufficient to permit bi-stability of a single component solid/melt system.

We have shown that the bi-stability of the steady states can manifest on a spatial level as existence of travelling front solutions - melting or crystallisation fronts. A travelling wave reduction of the full system has provided a basis for a dynamical system formulation of the linearised stability problem, and we have used Evans function methods to study the stability of fronts with respect to both longitudinal and transverse perturbations. These results suggest that the fronts are stable in both cases. Physically this suggests that crystallisation (melting) fronts can propagate in a phase change sample, and the stability calculations imply that they should be experimentally observable. On the other hand, the stability of these fronts indicates that even though the model at hand can be used for studying the processes of crystallisation and melting, it cannot be applied for the analysis of interface instability and dendritic growth. The reason for this is that in the evolution equation for the temperature the term describing latent heat production at the interface has been neglected in comparison with other contributions. However, given different material properties, the inclusion of latent heat in the thermal equation may well induce front instabilities.

It is worth noting that our model considers only a simple radiative thermal heating effect such as is used in data storage applications. The heating is 
governed by an absorption rate which is different for the solid and molten phases; the variation of this with wavelength and intensity of illuminating light may make possible the control of crystallisation fronts and therefore prove to be very useful in practice.

An interesting and important application of this work would be in the context of so-called explosive crystallisation within phase change materials. Some recent studies of this phenomenon, both theoretical and experimental in nature, have revealed the existence of unexpectedly high velocities of the fronts separating amorphous and crystalline regions $[22,26]$. The models adopted to date have neglected the difference in response of different phases to external heat sources but have concentrated instead on heat loss to surrounding substrates as well as latent heat release at the interface. An extension of the current phase field model could incorporate the latent heat release at the interface between amorphous and crystalline zones and thereby lead to a better understanding of explosive crystallisation.

\section{Acknowledgements}

This work was supported by EPSRC Grant GR/S31662/01.

\section{References}

[1] J.C. Alexander, R. Gardner, C.K.R.T. Jones, A topological invariant arising in the stability analysis of travelling waves, J. Reine Angew. Math. 410 (1990) $167-212$.

[2] L. Allen, T.J. Bridges, Numerical exterior algebra and the compound matrix method, Numer. Math. 92 (2002) 197-232.

[3] D.M. Anderson, A model for diffusion-controlled solidification of ternary alloys in mushy layers, J. Fluid Mech. 483 (2003) 165-197.

[4] N.J. Balmforth, R.V. Craster, S.J.A. Malham, Unsteady fronts in an autocatalytic system, Proc. R. Soc. London A 455 (1999) 1401-1433.

[5] K.B. Blyuss, P. Ashwin, A.P. Bassom, C.D. Wright, Master-equation approach to the study of phase-change processes in data storage media, Phys. Rev. E 72 (2005) 011607.

[6] K.B. Blyuss, T.J. Bridges, G. Derks, Transverse instability and its long-term development for solitary waves of the $(2+1)$-dimensional Boussinesq equation, Phys. Rev. E 67 (2003) 056626. 
[7] T.J. Bridges, G. Derks, G. Gottwald, Stability and instability of solitary waves of the fifth-order KdV equation: a numerical framework, Physica D 172 (2002) 190-216.

[8] G. Caginalp, W. Xie, Phase-field and sharp-interface alloy models, Phys. Rev. E 48 (1993) 1897-1909.

[9] J.W. Evans, Nerve axon equations IV. The stable and unstable impulse, Indiana Univ. Math. J. 24 (1975) 1169-1190.

[10] D.L. Feltham, M.G. Worster, Similarity solutions describing the melting of a mushy layer, J. Cryst. Growth 208 (2000) 746-756.

[11] S. Gatti, M. Graselli, V. Pata, Exponential attractors for a conserved phase-field system with memory, Physica D 189 (2004) 31-48.

[12] V. Gubernov, G.N. Mercer, H.S. Sidhu, R.O. Weber, Evans function stability of combustion waves, SIAM J. Appl. Math. 63 (2003) 1259-1275.

[13] A. Karma and W.-J. Rappel, Qualitative phase-field modeling of dendritic growth in two and three dimensions, Phys. Rev. E 57 (1998) 4323-4349.

[14] D. Kashchiev, Nucleation, Butterworth-Heinemann, Oxford, 2000.

[15] R. Kobayashi, Modelling and numerical simulations of dendritic crystal growth, Physica D 63 (1993) 410-423.

[16] R. Kobayashi, J.A. Warren, W.C. Carter, Vector-valued phase field model for crystallization and grain boundary formation, Physica D 119 (1998) 415-423.

[17] M. Libera, M. Chen, Multilayered thin-film materials for phase-change erasable storage, MRS Bull. 15 (1990) 40-45.

[18] A.E. Lobkovsky, J.A. Warren, Phase field model of premelting of grain boundaries, Physica D 164 (2002) 202-212.

[19] W.W. Mullins, R.F. Sekerka, Morphological instability of a particle growing by diffusion or heat flow, J. Appl. Phys. 34 (1963) 323-329.

[20] B.S. Ng, W.H. Reid, An initial-value method for eigenvalue problems using compound matrices, J. Comp. Phys. 30 (1979) 125-136.

[21] N. Ohshima, Crystallization of germanium-antimony-tellurium amorphous thin films sandwiched between various dielectric protective films, J. Appl. Phys. 79 (1996) 8357-8363.

[22] M. Okuda, H. Inaba, S. Usuda, Explosive crystallization in eutectic materials of phase change optical memory, MRS Symp. Proc. 803 (2004) 225-232.

[23] R.L. Pego, P. Smereka, M.I. Weinstein, Oscillatory instability of traveling waves for a KdV-Burgers equation, Physica D 67 (1993) 45-65.

[24] H.G. Rotstein, A.I. Domoshnitsky, A. Nepomnyashchy, Front motion for phase transitions in systems with memory, Physica D 146 (2000) 137-149. 
[25] G. Ruitenberg, A.K. Petford-Long, R.C. Doole, Determination of the isothermal nucleation and growth parameters for the crystallization of thin $\mathrm{Ge}_{2} \mathrm{Sb}_{2} \mathrm{Te}_{5}$ films, J. Appl. Phys. 92 (2002) 3116-3123.

[26] W. van Saarloos, J.D. Weeks, Surface undulations in explosive crystllization: a thermal instability, Phys. Rev. Lett. 51 (1983) 1046-1049.

[27] B. Sandstede, Stability of travelling waves, in Handbook of Dynamical Systems (ed. B. Fiedler), Elsevier, Amsterdam (2002).

[28] T. Schulze, M.G. Worster, Weak convection, liquid inclusion and the formation of chimneys in mushy layers, J. Fluid Mech. 388 (1999) 197-215.

[29] S. Senkader, C.D. Wright, Models for phase-change of $\mathrm{Ge}_{2} \mathrm{Sb}_{2} \mathrm{Te}_{5}$ in optical and electrical memory devices, J. Appl. Phys. 95 (2004) 504-511.

[30] J. Swinton, J. Elgin, Stability of travelling pulse to a lase equation, Phys. Lett. A 145 (1990) 428-433.

[31] J. Tominaga, T. Kikukawa, M. Takahashi, T. Kato, T. Aoi, Optical phase change discs without bulk laser initialization and a quick bulk initialization structure, Jpn. J. Appl. Phys. 36 (1997) 3598-3601.

[32] V. Weidenhof, I. Friedrich, S. Ziegler, M. Wuttig, Laser induced crystallization of amorphous $\mathrm{Ge}_{2} \mathrm{Sb}_{2} \mathrm{Te}_{5}$ films, J. Appl. Phys. 89 (2001) 3168-3176.

[33] A.A. Wheeler, W.J. Boettinger, G.B. McFadden, Phase-field model for isothermal phase transitions in binary alloys, Phys. Rev. A 45 (1992) 74247439 .

[34] A.A. Wheeler, B.T. Murray, R.J. Schaefer, Computation of dendrites using a phase field model, Physica D 66 (1993) 243-262.

[35] M.G. Worster, Convection in mushy layers, Ann. Rev. Fluid Mech. 29 (1997) $91-122$.

[36] M.G. Worster, Solidification of fluids, in Perspectives in fluid dynamics: a collective introduction to current research (eds. G.K. Batchelor, H.K. Moffatt \& M.G. Worster), 393-446, Cambridge University Press, Cambridge (2000).

[37] N. Yamada, Potential of Ge-Sb-Te phase-change optical discs for high-data-rate recording, Proc. SPIE 3109 (1997) 28-37. 